\title{
Desarrollo y validación de un patrón visual para la evaluación del color de la carne de bovino en México
}

Sara Salinas Labra ${ }^{a}$

María Salud Rubio Lozano ${ }^{b}$

Diego Braña Varela ${ }^{c}$

Rubén Danilo Méndez Medina ${ }^{b}$

Enrique Jesús Delgado Suárez ${ }^{b^{*}}$

a Zoetis México.

${ }^{\mathrm{b}}$ Universidad Nacional Autónoma de México, Facultad de Medicina Veterinaria y Zootecnia, Ciudad de México, México.

c Elanco Animal Health.

*Autor de correspondencia: enriquedelgado.suarez@gmail.com

\section{Resumen:}

El objetivo fue desarrollar una escala visual para la evaluación del color en carne de bovino. Se analizaron 1,165 lomos, a las 24 h post mortem, en cuatro rastros de la República Mexicana. En cada muestra se evaluó el color con ayuda de un patrón visual y con un espectrofotómetro (escala CIELAB), tomándose una fotografía de cada lomo. Por el método visual se identificaron siete categorías, (de rojo muy pálido a rojo muy oscuro) y las variables instrumentales del color $\left(\mathrm{L}^{*}, \mathrm{a}^{*}, \mathrm{~b}^{*}, \mathrm{C}^{*} \mathrm{y} \mathrm{h}^{*}\right)$ se usaron para generar modelos de predicción de las categorías visuales. La escala se construyó utilizando $\mathrm{L}^{*}$ como único predictor, pues este modelo explicó >90 \% de la variación observada. El patrón se ilustró con fotos de las muestras con valor de $\mathrm{L}^{*}$ dentro del intervalo de confianza al $95 \%$ de la media en cada categoría, desde rojo muy pálido $\left(48.1<\mathrm{L}^{*}<48.8\right)$ hasta rojo muy oscuro $\left(32.7<\mathrm{L}^{*}<33.4\right)$. La diferencia total de color entre categorías fluctuó entre 2.8 y 5.5, lo que sugiere que éstas son 
diferenciables a simple vista. La escala se validó mediante pruebas con un panel sensorial entrenado y otro de consumidores. Los jueces entrenados calificaron correctamente las muestras en el $92.6 \%$ de las evaluaciones. En carne con apariencia de corte oscuro (CO), el panel entrenado tuvo $100 \%$ de aciertos y el de consumidores $85.3 \%$. El patrón visual propuesto se sustenta en mediciones instrumentales y mostró ser técnicamente viable para la evaluación del color en carne de bovino por personal entrenado y por consumidores.

Palabras clave: Carne de res, Bovino, Calidad, Color, Visual, Instrumental, Patrón.

Recibido: 05/12/2018

Aceptado: 30/07/2019

\section{Introducción}

El color de la carne fresca se encuentra dentro de los principales atributos de calidad que influyen en la decisión de compra del consumidor ${ }^{(1,2)}$. Es un hecho que los defectos en la coloración de la carne causan cuantiosas pérdidas económicas, debido a que provocan penalizaciones en su precio $^{(3-5)}$. Por ello, el color es uno de los principales atributos empleados para evaluar la calidad de canales y de carne en países que son grandes comercializadores, como Estados Unidos, Japón, Canadá y Australia ${ }^{(6-9)}$. Estos esquemas de valoración se realizan con la ayuda de escalas visuales, las cuales están altamente correlacionadas con la decisión de compra del consumidor ${ }^{(10)}$. Además, la detección temprana de defectos en el color permite segregar la carne con apariencia indeseable y redirigirla a procesos de manufactura en los que este atributo tiene menor relevancia ${ }^{(7)}$.

La razón de que se desarrollen patrones visuales para el mismo fin en diferentes naciones, radica en que el color de la carne es un fenómeno multifactorial. Por ejemplo, se conoce que factores como la raza, el sistema de producción, la dieta, así como el manejo previo y posterior a la matanza, los cuales varían de un país a otro, constituyen importantes fuentes de variación en el color de la carne ${ }^{(11,12)}$. Por tanto, el empleo de estas herramientas por parte de la industria cárnica debe basarse en evidencias científicas generadas a partir del ganado local. Lo anterior cobra especial relevancia en el ganado bovino, especie que suele presentar una alta variación en sus atributos de calidad ${ }^{(13)}$.

En México, la ganadería bovina de carne está entre las actividades pecuarias de mayor importancia económica ${ }^{(14)}$. Además, la carne de res goza de amplia popularidad en el país, con un consumo per capita anual de $17.40 \mathrm{~kg}^{(15)}$; sin embargo, no se cuenta con herramientas científicamente sustentadas para la segregación de la carne según el color. La norma 
mexicana de clasificación de canales incluye al color como uno de los atributos que determinan el grado de calidad de éstas ${ }^{(16)}$. Sin embargo, propone su evaluación a partir de una escala de colores sólidos de apenas tres niveles, del sistema pantone, que asocia con carne de color rojo-cereza, rojo intenso y rojo oscuro. Tal escala no es representativa de toda la gama de tonalidades que puede tener la carne de bovino y tampoco describe la apariencia asociada con defectos de calidad, como el corte oscuro (CO). Además, no toma en cuenta que la superficie de la carne es irregular, con fibras musculares en diferentes direcciones, presencia de tejido conectivo y grasa entreverada. Es por ello que el empleo de patrones fotográficos se considera una mejor alternativa para la evaluación subjetiva del color de la carne $^{(17)}$.

Aunque actualmente existen algunas iniciativas privadas y se han realizado varios estudios de alcance regional ${ }^{(18-22)}$, hasta el momento no se ha desarrollado un patrón visual que sirva de referencia para la industria nacional. Tal carencia representa una desventaja comercial para los productores locales, que pueden recibir penalizaciones en el precio de la carne con base en valoraciones subjetivas por parte de sus clientes. Por tanto, el objetivo del presente trabajo es desarrollar y validar un patrón para la evaluación visual del color de la carne de bovino a escala industrial en México. Con ello, se espera contribuir al ordenamiento del mercado, a mejorar la comunicación entre los distintos eslabones de la cadena de valor, así como a generar un método de evaluación subjetivo del color de la carne, científicamente sustentado, que subsane las deficiencias que presenta el sistema considerado en la normatividad vigente

\section{Material y métodos}

\section{Universo muestral}

Se utilizó un plan de muestreo no probabilístico, en el que se aplicaron los siguientes criterios para seleccionar los rastros que participaron en el estudio: 1) empresas ubicadas en alguna de las tres zonas ecológico-ganaderas de México (árida y semi-árida, trópico húmedo y trópico seco), 2) que las medias canales se cortaran entre la doceava y treceava costillas, 3) que fuera un rastro Tipo Inspección Federal (TIF) y 4) que existieran las condiciones técnicas de iluminación, espacio, acceso a los cortes primarios y área de cortes con temperatura de refrigeración de $6-8^{\circ} \mathrm{C}$ para realizar los trabajos.

Con base en estos criterios, se seleccionaron cuatro rastros TIF, ubicados en Baja California, Sinaloa, Querétaro y Tabasco, en los que se analizó un total de 1,165 muestras (Figura 1). Como la carne fue sometida a un tiempo de oxigenación de 30 min antes de evaluar el color, esto obligaba a trabajar a un ritmo mucho más lento en comparación con el flujo de proceso. Por ello, no fue posible analizar todas las canales procesadas en un día. Entonces, se fijó 
como meta tomar al menos 170 canales por rastro, que fue el tamaño de muestra mínimo determinado, considerando un nivel de confianza del 95\%, una precisión de 0.5 unidades en las variables instrumentales del color y una varianza de 10.69 , estimada a partir de pruebas preliminares. Así, mediante la fórmula $n=\frac{Z_{\alpha}^{2} * S^{2}}{d^{2}}$, se obtuvo un tamaño de muestra de 164, el cual se redondeó a 170. Sin embargo, con el fin de obtener la mayor variación posible, en cada establecimiento se analizaron todos los lomos que fue posible recolectar en un turno de 8 h durante un período de 3 o 4 días. Por ello, el tamaño de muestra real por rastro fluctuó entre 172 y 405, según el volumen de matanza en cada establecimiento.

Figura 1: Número de muestras de lomo analizadas en rastros TIF de cuatro estados de la República Mexicana entre noviembre de 2012 y julio de 2013

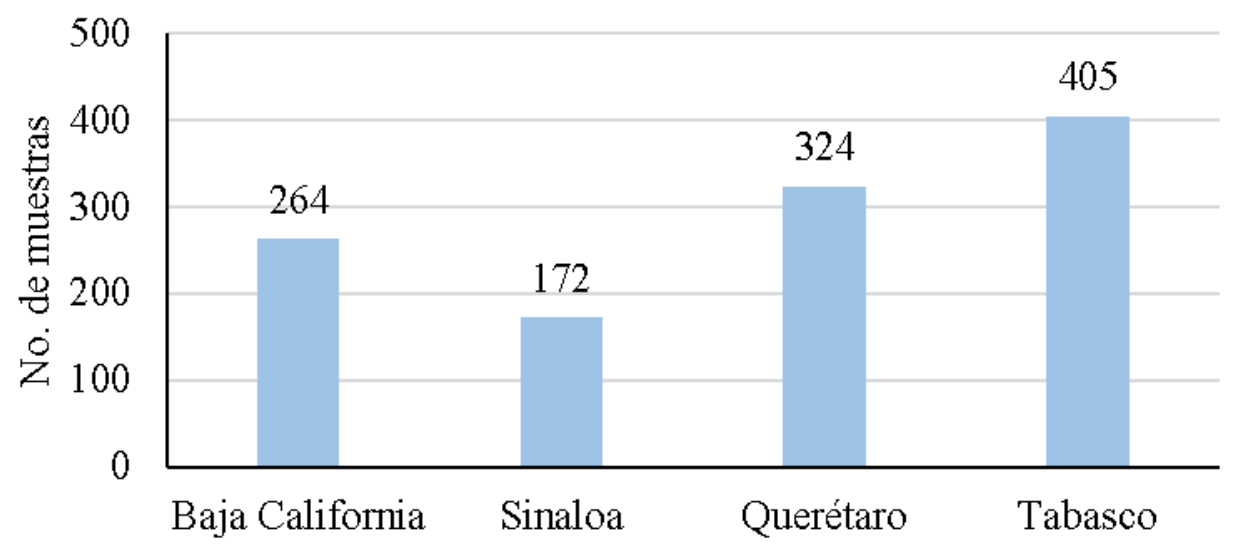

La población de animales de la que se obtuvieron los datos estaba constituida, en cerca de un $80 \%$, por machos enteros sin castrar y el porcentaje restante por hembras. Como la investigación se realizó en rastros, no siempre fue posible conocer la edad al sacrificio. No obstante, en una muestra de alrededor de 300 animales en que se disponía de este dato, el $86 \%$ tenía 24 meses o menos. En general, cabe esperar que estas características fueran predominantes en la muestra estudiada, tomando en cuenta reportes previos que documentan la preponderancia de toros jóvenes en la población de matanza de bovinos de carne en México $^{(23)}$.

\section{Medición del color}

Las mediciones de color, tanto visuales como instrumentales, se realizaron según los lineamientos de la Asociación Americana de Ciencia de la Carne ${ }^{(24)}$. Las lecturas se realizaron a las $24 \mathrm{~h}$ post mortem, en el área de corte y deshuese de cada rastro TIF, con temperatura ambiente controlada de 6 a $8{ }^{\circ} \mathrm{C}$. Para ello, se recolectaron los lomos completos (músculo Longissimus dorsi), recién separados de la canal y con una temperatura no mayor a $3{ }^{\circ} \mathrm{C}$. Las piezas así seleccionadas se dejaron reposar durante $30 \mathrm{~min}$ en una mesa de acero 
inoxidable, con el área del ojo de la chuleta entre las costillas doceava y treceava expuesta al aire, para permitir una óptima oxigenación de la mioglobina previo a las lecturas.

La evaluación visual del color se realizó bajo condiciones estandarizadas. Se utilizó como fuente de luz una lámpara incandescente Osram de calidad fotográfica, con una intensidad de 150 candelas (1,614 luxes) y una temperatura del color de $3,200^{\circ} \mathrm{K}$, colocada en un ángulo de $45^{\circ}$ con respecto a la superficie de la chuleta. A cada chuleta evaluada se le tomó una foto de alta resolución, con ayuda de un fotógrafo profesional, que empleó para este fin una cámara Nikon D300S de 12 megapixeles, con lente Zoom de 24-70 f. 2.8 marca Sigma. Las fotos se tomaron contra un fondo negro, para eliminar diferencias de color asociadas con la superficie de la carne. Las fotografías se tomaron con el fin de contar con imágenes ilustrativas de las diferentes tonalidades de la carne, mismas que podrían ser incluidas en la escala visual de color que se estaba desarrollando. Posteriormente, el color se evaluó de manera visual, con el patrón para carne de bovino de Estados Unidos (Figura 2), que consta de ocho niveles ${ }^{(9)}$. Esta actividad se realizó por un investigador experimentado en evaluación de canales bajo el esquema norteamericano, con el fin de tener una referencia preliminar sobre el número de categorías visuales que podrían presentarse en la muestra estudiada.

Figura 2: Escala visual para la evaluación del color en canales de bovinos desarrollada en Estados Unidos de América ${ }^{(9)}$
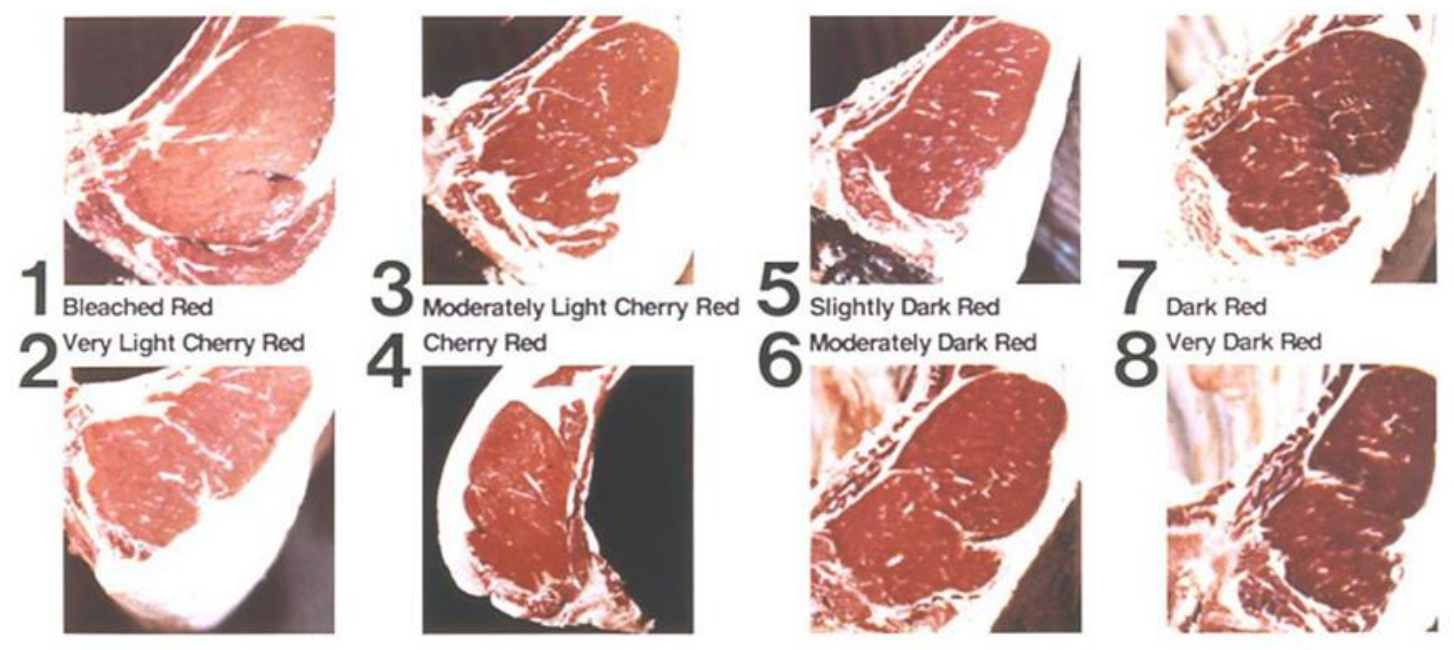

Para las mediciones instrumentales se empleó un espectrofotómetro Hunter MiniScan EZ modelo 4500L, con geometría 45/0 y un tamaño de apertura del puerto de $25 \mathrm{~mm}$ (Hunter Associates Laboratory, Inc, Reston, Virginia, Estados Unidos). El instrumento se configuró con iluminante $\mathrm{A}$, observador a $10^{\circ}$ y el componente especular excluido. Además, se operó en modo remoto, por medio del programa OnColor QC Lite, versión 6 (CyberChrome, Inc., New Paltz, New York, Estados Unidos), para la captura de los datos en computadora. Se realizaron calibraciones previo al inicio de las mediciones y posteriormente cada 100 lecturas 
o después de 1 hora (lo que ocurriera primero), empleando la trampa negra y el azulejo blanco suministrados por el fabricante. En cada chuleta se tomaron cuatro lecturas, las cuales se promediaron para obtener los valores de luminosidad $\left(\mathrm{L}^{*}\right)$, intensidad de rojo ( $\left.\mathrm{a}^{*}\right)$, intensidad de amarillo $\left(\mathrm{b}^{*}\right)$, tonalidad $\left(\mathrm{h}^{*}\right)$ e índice de satuación $\left(\mathrm{C}^{*}\right)$ de cada chuleta en la escala CIELAB. Junto con estos datos se recolectaron también las curvas espectrales, mismas que constituyen la huella de color y que fueron necesarias para realizar la impresión profesional de la escala.

\section{Análisis de datos y conformación de la escala visual}

Para el análisis estadístico se empleó el software Statgraphics XV Centurion para Windows (Statpoint, Inc., The Plains, Virginia, Estados Unidos). Se compararon las medias de los valores instrumentales del color entre las categorías de calidad asignadas visualmente. Para ello, se realizó un análisis de varianza (ANOVA) de clasificación simple, mediante el procedimiento de Modelos Lineales Generalizados (MLG). Como se tenía un número diferente de observaciones por nivel, cuando se encontraron diferencias significativas, las medias se discriminaron mediante el procedimiento de comparación múltiple de Bonferroni. Para la construcción de la escala, se probaron distintos modelos de predicción, también con el procedimiento MLG, utilizando la categoría visual como variable dependiente y diferentes combinaciones de las variables instrumentales y sus interacciones como variables explicativas (ver Cuadro S1 en información suplementaria). Entre los modelos generados, se eligió el que explicara el mayor porcentaje de la variación observada entre categorías visuales, que resultó ser el que utiliza $\mathrm{L}^{*}$ como única variable explicativa. Por ello, se construyeron los intervalos de confianza al $95 \%$ de $\mathrm{L}^{*}$ dentro de cada categoría visual. Para ilustrar la escala, se seleccionaron las fotos que correspondían a chuletas cuyos valores de L* se encontraban dentro del intervalo de confianza de la media en cada categoría.

Por último, era necesario determinar si las categorías así definidas podrían ser diferenciables de manera visual. Para este fin, se calculó la diferencia total de color $\left(\Delta E^{*}{ }_{\mathrm{ab}}\right)$, la cual se define como la suma de las diferencias modulares de $L^{*}, a^{*}$ y $b^{*}$ entre dos muestras: $\left(\Delta E^{*}{ }_{\mathrm{ab}}=\right.$ $\sqrt{\Delta \mathrm{L}^{*}+\Delta \mathrm{a}^{2}+\Delta \mathrm{b}^{*}}$ ). A mayor $\Delta E^{*}$ ab, más fácil es distinguir la diferencia de color entre dos muestras de manera visual. No obstante, por lo general muestras con valores de $\Delta E^{*}{ }_{\mathrm{ab}}>2$ unidades se consideran fácilmente diferenciables a simple vista ${ }^{(25)}$.

\section{Experimento de validación preliminar}

Con el fin de validar la escala visual previamente generada, el patrón se desafió a escala industrial, bajo las condiciones que a continuación se describen. Se trabajó en un rastro que no participó en el muestreo inicial. Para la validación, se conformó un panel sensorial 
entrenado, con seis personas familiarizadas con el patrón de color propuesto. Entre los candidatos a conformar el panel, los integrantes fueron seleccionados a partir de la prueba de Farnsworth-Munsell (http://www.color-blindness.com/farnsworth-munsell-100-hue-colorvision-test/\#prettyPhoto), la cual permite descartar a las personas con deficiencias en la apreciación del color. El panel se conformó únicamente con jueces que tuvieron un resultado de deficiencia en la visión del color de "ninguna" o "ligera" (0 a 70 puntos). Se partió de un grupo inicial de 20 candidatos, los cuales se seleccionaron con base en lineamientos generalmente aceptados para la planificación y selección de jueces entrenados ${ }^{(26)}$.

Como resultado de este proceso, el panel sensorial quedó conformado por seis integrantes, con los cuales se realizaron seis sesiones de evaluación, en las que cada juez evaluó 6 muestras de carne (2 de carne de color normal (N), 2 de CO moderada y 2 de CO extrema), para un total de 36 mediciones por juez. Para las evaluaciones se utilizó una escala estructurada de $10 \mathrm{~cm}$ de longitud, acotada desde rojo muy pálido ( $\mathrm{L}^{*}=50$ ) hasta rojo muy oscuro $\left(\mathrm{L}^{*}=30\right)$ y que fue previamente explicada a los jueces:

Rojo muy pálido

$\left(\mathrm{L}^{*}=50\right)$
Rojo muy oscuro

$\left(\mathrm{L}^{*}=30\right)$

Según la escala visual desarrollada, la carne CO extrema tiene valores de $\mathrm{L}^{*}$ entre 30 y 34 ; la CO moderada, de 35-37 y la carne N, 38 o más. Se pidió a cada juez que marcara con una cruz en la escala, para indicar el color que correspondía a la muestra evaluada. La posición señalada por los jueces se midió con una regla para obtener el valor de $\mathrm{L}^{*}$ estimado. Los datos se analizaron mediante un análisis de varianza de 3 factores (juez, muestra, sesión y sus interacciones). Esto permitió verificar que la única fuente de variación significativa en las evaluaciones era la muestra. Por último, se realizó un análisis de correlación entre los valores de $\mathrm{L}^{*}$ estimados a partir de la calificación del color realizada por los jueces y los valores de $\mathrm{L}^{*}$ reales medidos con el espectrofotómetro. Para considerar la escala visual como válida para evaluar el color de la carne de bovino, se requirió obtener un poder discriminatorio significativo de los jueces, una correlación significativa de alta magnitud entre los valores de $\mathrm{L}^{*}$ reales de las muestras y los valores de $\mathrm{L}^{*}$ estimados por los jueces, así como un porcentaje de aciertos $\geq 80 \%$, por parte de los jueces, al asignar la categoría de color a cada muestra. En trabajos previos con jugo de naranja y con vinos, las diferencias visuales de las muestras se han validado con un porcentaje de aciertos mucho menor del panel sensorial (ej. $>50 \%)^{(27,28)}$. No obstante, en la presente investigación se utilizó un punto de quiebre más alto, con el fin de determinar si la escala visual funcionaba para la mayor parte de las personas, razón por la cual también se solicitó a un panel de personas no entrenadas $(\mathrm{n}=6)$, que dictaminaran si existía diferencia entre muestras de carne $\mathrm{N}$ y CO. Ello con el fin de comprobar la confiabilidad de la escala, en caso de ser utilizada por personas sin entrenamiento en evaluación del color. Las muestras fueron seleccionadas y presentadas bajo las mismas condiciones descritas anteriormente para el panel entrenado. No obstante, al panel 
no entrenado solamente se le presentaron pares de muestras y se pidió a los jueces que eligieran la muestra que preferían según el color.

\section{Resultados}

\section{Definición de categorías visuales y su relación con mediciones instrumentales}

La evaluación visual permitió identificar en la muestra estudiada siete de las ocho clases representadas en el patrón norteamericano. Lo anterior debido a que ninguna de las muestras tuvo apariencia similar a la categoría 5 (ligeramente rojo oscuro). La distribución de las muestras en cada una de las siete clases identificadas se presenta en la Figura 3.

Figura 3: Distribución de muestras $(n=1,165)$ en cada una de las categorías visuales identificadas con ayuda del patrón norteamericano para la evaluación del color en carne de bovino (la descripción de cada categoría es igual que en la Figura 2).

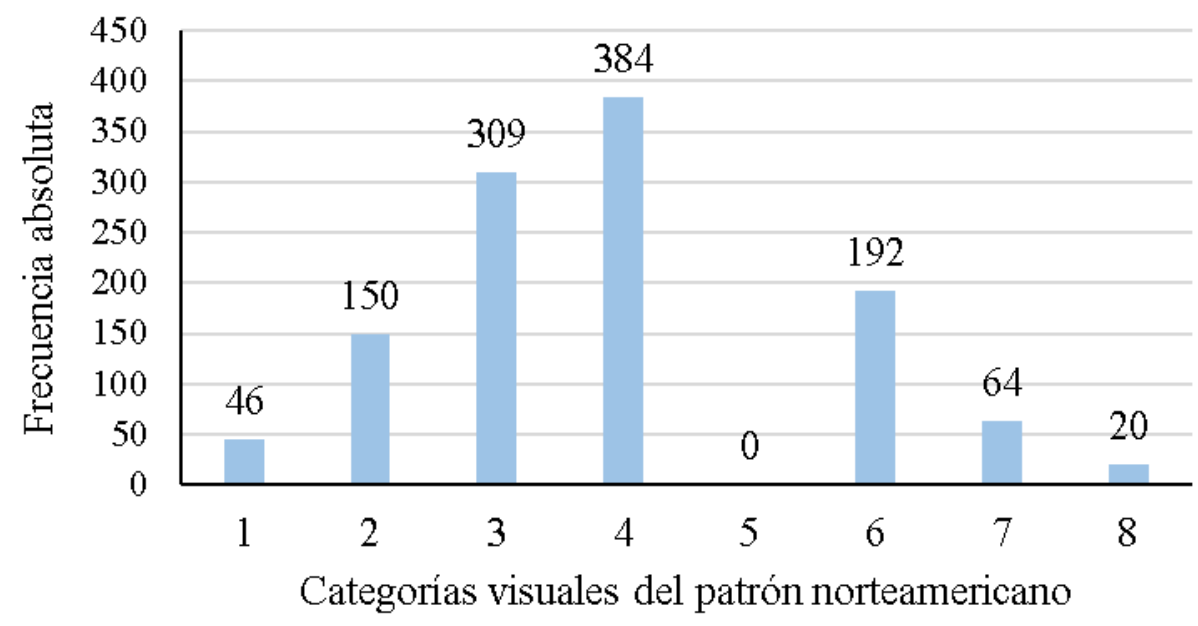

La proporción de carne asociada con defectos de calidad (categorías 7 y 8, que representan diferentes grados de la apariencia $\mathrm{CO}$ ) fue relativamente baja (7\%). Por el contrario, más del $70 \%$ de las muestras se ubicó entre las categorías 2 a 4, apariencia que típicamente se asocia con carne de calidad normal.

Por otra parte, el ANOVA resultó significativo $(P<0.0001)$ para todas las variables instrumentales del color (Cuadro 1). No obstante, $L^{*}$ fue la única que presentó diferencias significativas entre las medias de todas las categorías. Además, el ANOVA de L* fue el único que tuvo un alto coeficiente de determinación $\left(\mathrm{R}^{2}=0.9171\right)$, mientras que en las otras variables osciló entre 0.2744 y 0.4284 . 
Cuadro 1: Medias de mínimos cuadrados de las variables instrumentales del color en carne de cada una de las categorías visuales identificadas en la muestra

\begin{tabular}{|c|c|c|c|c|c|c|c|c|c|}
\hline \multirow[b]{2}{*}{ Variable } & \multicolumn{7}{|c|}{ Categorías visuales } & \multirow[b]{2}{*}{$\mathbf{E E} \pm^{1}$} & \multirow[b]{2}{*}{$\mathbf{R}^{2}$} \\
\hline & $\mathbf{1}$ & 2 & 3 & 4 & 6 & 7 & 8 & & \\
\hline $\mathrm{n}$ & 30 & 62 & 283 & 264 & 314 & 144 & 68 & & \\
\hline $\mathrm{L}^{*}$ & $48.8^{\mathrm{a}}$ & $46.7^{\mathrm{b}}$ & $44.4^{\mathrm{c}}$ & $42.1^{\mathrm{d}}$ & $40.1^{\mathrm{e}}$ & $37.7^{\mathrm{f}}$ & $34.8^{\mathrm{g}}$ & $0.94 * * *$ & 0.9171 \\
\hline$a^{*}$ & $27.4^{\mathrm{a}}$ & $27.8^{\mathrm{a}}$ & $27.5^{\mathrm{a}}$ & $27.1^{\mathrm{ab}}$ & $26.3^{\mathrm{b}}$ & $25.1^{\mathrm{c}}$ & $22.6^{\mathrm{d}}$ & $2.04 * * *$ & 0.2744 \\
\hline$b^{*}$ & $20.1^{\mathrm{a}}$ & $20.2^{\mathrm{a}}$ & $19.1^{\mathrm{b}}$ & $18.3^{\mathrm{c}}$ & $17.3^{\mathrm{d}}$ & $16.0^{\mathrm{e}}$ & $13.5^{\mathrm{f}}$ & $2.12 * * *$ & 0.3522 \\
\hline $\mathrm{C}^{*}$ & $34.1^{\mathrm{a}}$ & $34.3^{\mathrm{a}}$ & $33.4^{\mathrm{ab}}$ & $32.7^{\mathrm{b}}$ & $31.5^{\mathrm{c}}$ & $29.8^{\mathrm{d}}$ & $26.3^{\mathrm{e}}$ & $2.21 * * *$ & 0.3082 \\
\hline $\mathrm{h}^{*}$ & $36.3^{\mathrm{a}}$ & $35.9^{\mathrm{a}}$ & $34.7^{\mathrm{b}}$ & $33.9^{c}$ & $33.2^{\mathrm{d}}$ & $32.4^{\mathrm{e}}$ & $30.7^{\mathrm{f}}$ & $1.41 * * *$ & 0.4284 \\
\hline
\end{tabular}

Aunque se probaron otros modelos de predicción (ver Cuadro S1 en información suplementaria), ninguno de ellos mejoró el que se obtuvo con $\mathrm{L}^{*}$ como única variable explicativa y, por tanto, fueron descartados. Por ello, se utilizaron los intervalos de confianza al $95 \%$ de la media de $\mathrm{L}^{*}$ como criterio para seleccionar las fotos representativas de cada una de las categorías visuales que conformaron la escala de color desarrollada (Figura 4). Un rasgo notorio de esta última es que la apariencia de la carne en algunas categorías adyacentes es muy parecida. Por ejemplo, las categorías 1 y 2 son representativas de carne pálida. De igual forma, las categorías 3 y 4 son representativas del tono rojo-cereza brillante, que suele ser el más atractivo para los consumidores en el punto de venta. Por ello, en un principio se consideró fusionar ambos pares de categorías en una sola. Sin embargo, el $\Delta E^{*}$ ab entre estos pares de categorías fue de casi 3 unidades CIELAB, lo que indica que son claramente diferenciables entre sí a simple vista. Por tanto, en lugar de fusionarlas, lo que se hizo fue utilizar una denominación que permitiera al usuario identificar que ambas categorías están asociadas con carne de tonalidad similar. Así, las categorías 1 y 2 fueron renombradas como $1 \mathrm{~A}$ y $1 \mathrm{~B}$, respectivamente, mientras que 3 y 4 fueron denominadas $2 \mathrm{~A}$ y $2 \mathrm{~B}$. Estos ajustes obligaron a modificar también la denominación de las categorías restantes (5, 6 y 7), quedando éstas como 3, 4 y 5, cuya apariencia es claramente diferenciable entre sí, con un $\Delta E^{*}{ }_{\text {ab }}$ entre estas clases mucho mayor (3.3 a 5.5 unidades CIELAB). 
Figura 4: Escala visual para la evaluación del color de la carne de bovino en México

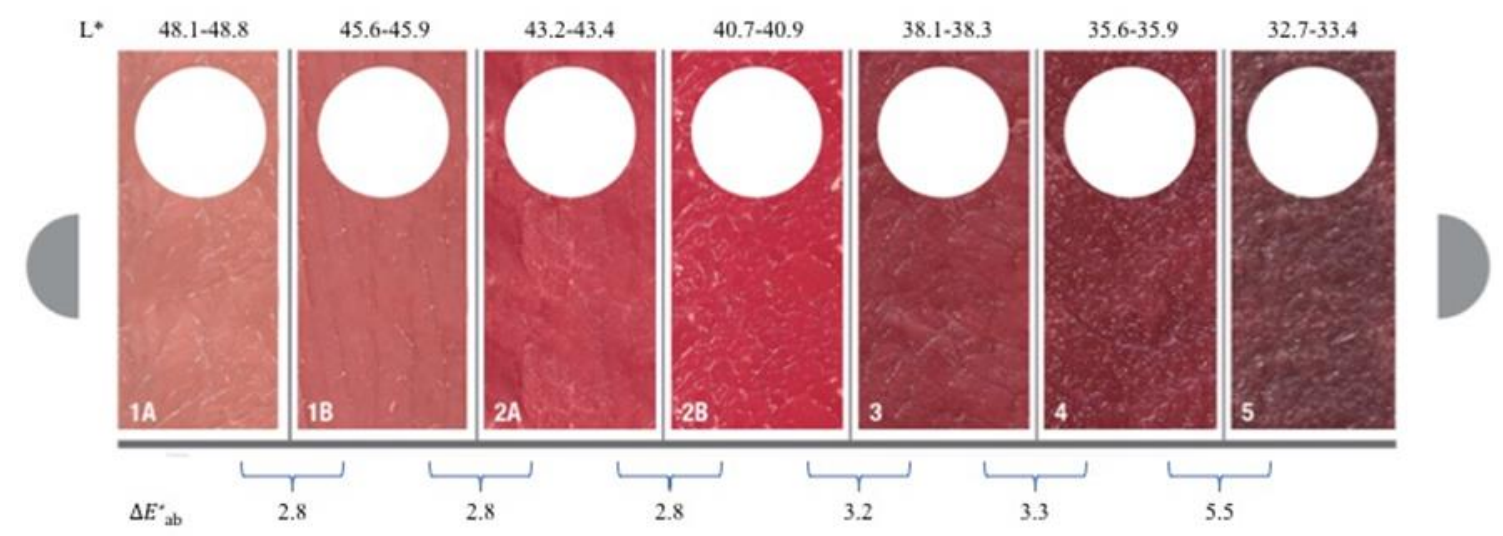

Los valores de $\mathrm{L}^{*}$ que se indican corresponden al intervalo de confianza al $95 \%$ de la media para cada categoría. En la parte inferior, se señalan las diferencias totales de color $\left(\Delta E^{*}{ }_{\mathrm{ab}}\right)$ entre categorías adyacentes.

\section{Validación preliminar de la escala descriptiva en condiciones industriales}

El primer criterio de validación de la escala de color fue que el panel sensorial detectara las diferencias entre las muestras. En este sentido, se obtuvieron resultados satisfactorios, pues tanto los jueces en lo individual, como el panel como grupo, mostraron un poder discriminatorio suficiente (ANOVA significativo, $P<0.0001$ ) para detectar las diferencias entre las muestras. Además, se comprobó que el único factor que incidía significativamente $(P=0.0002)$ en la puntuación de los jueces era la muestra (ver Cuadros S2 a S4 en información suplementaria).

La correlación entre los valores reales de $\mathrm{L}^{*}$ de las muestras y los asignados por los jueces (Figura 5), que fue el segundo criterio de validación, fue significativa y de alta magnitud $(\mathrm{r}=0.9338, P<0.0001)$. Estos resultados se corresponden con el porcentaje de aciertos por parte de los jueces, tercer y último criterio de validación de la escala, los cuales, en promedio, asignaron correctamente las muestras a la categoría visual del patrón en el $92.6 \%$ de las evaluaciones. En cuanto a detección de $\mathrm{CO}$, la escala mostró un desempeño aun mejor, pues las muestras que presentaban esta condición fueron correctamente asignadas en el $100 \%$ de los casos por todos los jueces. También se comprobó que es posible distinguir los diferentes grados del defecto CO, ya que los jueces entrenados sólo confundieron la CO moderada con la extrema en el $5 \%$ de las evaluaciones. Por último, en la evaluación realizada por el panel no entrenado, se comprobó la pertinencia de las categorías que describen el defecto CO. Lo anterior en virtud de que en el $85.3 \%$ de las evaluaciones realizadas por los panelistas no entrenados, se prefirió el color de la carne $\mathrm{N}$ sobre la CO. En general, los resultados de las pruebas sensoriales demuestran la viabilidad técnica de la escala de color propuesta, toda vez que tanto personal entrenado como consumidores fueron capaces de utilizarlo para dictaminar de manera correcta la apariencia de la carne. 
Figura 5: Análisis de regresión entre L* estimada por el panel sensorial entrenado y L* real de las muestras medido con el espectrofotómetro $(n=108)$

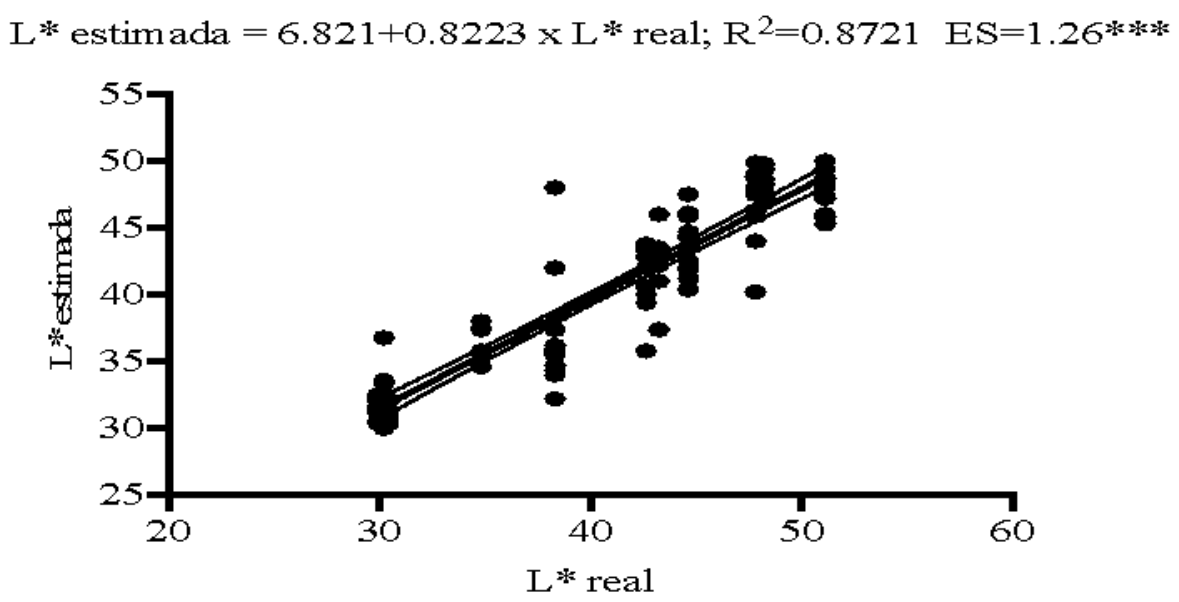

El modelo incluye los límites de predicción bilaterales. $* * * P<0.0001$.

\section{Discusión}

En esta investigación se presenta el primer patrón visual para la evaluación del color de la carne de bovino en México, sustentado científicamente con datos tomados en el ganado que se produce en el país. La escala desarrollada contiene categorías que describen carne de apariencia pálida, rojo-cereza, rojo fuerte, rojo oscuro y rojo muy oscuro. Estas tonalidades son claramente diferenciables de manera visual y, además, muestran una alta correlación con variables instrumentales del color, particularmente con L*. Esto coincide con lo observado en estudios anteriores, en los que $\mathrm{L}^{*}$ ha sido la variable mejor relacionada con la apariencia visual de la carne ${ }^{(29,30)}$.

En algunos estudios realizados en México, se ha utilizado la saturación del color $\left(\mathrm{C}^{*<30)}\right.$ como uno de los criterios para identificar carne $\mathrm{CO}^{(3)}$. Aunque en este estudio se utilizó un instrumento y condiciones de medición diferentes, se observaron también valores de $\mathrm{C}^{*}$ inferiores a 30 en carne con apariencia de cortes oscuros. No obstante, $C^{*}$ sólo explicó cerca del $30 \%$ de las diferencias entre los distintos niveles de la escala, mientras que para $\mathrm{L}^{*}$ el coeficiente de determinación fue superior al $90 \%$. De hecho, la alta correlación entre L* y la apariencia visual que se observó en este estudio sugiere que las mediciones instrumentales se pueden usar ya sea para orientar a los evaluadores que utilizan la escala visual, o bien, para sustituir el empleo de esta última en empresas que cuenten con tecnología instrumental para medir el color.

Por otra parte, las categorías o clases incluidas en la escala se pueden asociar con determinadas ventajas comerciales o con defectos de calidad. Por ejemplo, la carne 
representada en las categorías 1A y 1B posee una apariencia similar a la que se describe en carne pálida, suave y exudativa (PSE) ${ }^{(31)}$. Este defecto se asocia con propiedades funcionales deterioradas, especialmente con una reducida capacidad de retención de agua. Se sabe, no obstante, que el fenómeno PSE ocurre con muy poca frecuencia en la especie bovina y por lo general sólo se presenta cuando se aplica un enfriamiento muy lento de las canales ${ }^{(32)}$. Aun así, 46 unidades (3.9\%) de la muestra analizada presentaron una apariencia extremadamente pálida y otras $150(12.9 \%)$ se clasificaron como moderadamente pálidas. Probablemente esto se deba a deficiencias en la velocidad de enfriamiento de las canales, o a una muscularidad excesiva que limite o retrase la pérdida de calor y promueva una caída más rápida del $\mathrm{pH}$ muscular, condiciones que podrían ser comunes en muchos rastros TIF en México. No obstante, investigaciones recientes sugieren que el color rojo claro es percibido como indicador de calidad en carne de bovino por los consumidores mexicanos ${ }^{(33)}$, lo que implica que la carne fresca con esta apariencia no debe sufrir penalizaciones en precio.

Las siguientes dos categorías (2A y 2B), representan la apariencia típica (rojo-cereza brillante) que los consumidores aprecian en la carne fresca ${ }^{(4)}$. Al parecer, cabe esperar que una buena parte de la carne de bovino que se produce en el país cumple con esta demanda, ya que $60 \%$ de las muestras analizadas presentaron estas tonalidades. Por tanto, la identificación de este tipo de carne constituye un apoyo para explotar al máximo las ventajas competitivas que ofrece su favorable apariencia para las ventas al detalle.

La categoría 3, por su parte, describe la carne que se encuentra en el límite de calidad aceptable. Su apariencia es un poco más oscura, determinada por los valores más bajos de $\mathrm{L}^{*}$, lo que la pone en desventaja con respecto al rojo-cereza, que se asocia con animales más jóvenes. Es conocido que, a medida que los animales envejecen, la luminosidad de la carne se reduce, lo que resulta en una apariencia más oscura de esta ${ }^{(34)}$. No obstante, la categoría 3 guarda una diferencia total de color bastante segura (3-5 unidades CIELAB) con respecto a las categorías que describen el defecto $\mathrm{CO}$ (4 y 5).

Por último, las categorías 4 y 5 son representativas del defecto $\mathrm{CO}$, el cual provoca pérdidas millonarias a la industria y es, por mucho, a escala global, el defecto de calidad de mayor importancia en carne de bovino ${ }^{(7)}$. Aunque se han descrito diferentes grados de la condición $\mathrm{CO}$ (ej. clásica, ligera y atípica), estudios recientes han demostrado que todas ellas tienen en común una apariencia oscura indeseable y atributos de calidad deteriorados ${ }^{(5,35)}$, por lo que ameritan penalización económica. En México, se ha estimado que el valor de las canales CO se reduce en aproximadamente $85 \mathrm{USD}^{(3)}$. Aunque la proporción de unidades con apariencia $\mathrm{CO}$ en la muestra analizada es aparentemente baja (7\%), el impacto económico de esta tasa, si se presentara a escala nacional, puede representar millones de dólares, tomando en cuenta que la matanza anual es de 4 millones de cabezas, tan solo en rastros $\operatorname{TIF}^{(36)}$. Por ello, la descripción de carne $\mathrm{CO}$ en la escala propuesta es de gran relevancia, puesto que provee las bases para la detección temprana de este defecto, ofreciendo la posibilidad de segregar la 
carne defectuosa y, además, gestionar los procesos asociados con la matanza, para reducir su incidencia.

El cálculo de $\Delta E^{*}{ }_{\text {ab }}$ demostró que las categorías representadas en la escala pueden ser fácilmente diferenciadas de manera visual. En algunos estudios se ha sugerido que el ojo humano puede percibir diferencias en el color a partir de valores de $\Delta E^{*}{ }_{\text {ab }} \geq 1^{(25)}$, cifra muy inferior a las que se observaron en este trabajo entre las categorías visuales propuestas (2.8$5.5)$.

Por otra parte, la validación demostró la pertinencia de la estrategia seguida para desarrollar la escala, con una alta correlación entre las evaluaciones del panel sensorial entrenado y la variable predictiva utilizada $\left(\mathrm{L}^{*}\right)$. Sin duda, esto contribuyó a que más del $90 \%$ de los jueces entrenados asignaran correctamente la categoría visual a las muestras evaluadas. De igual forma, se comprobó que las categorías asociadas con $\mathrm{CO}$, que es el principal defecto de calidad en carne de bovino, son fácilmente identificadas tanto por personas entrenadas como por consumidores. Ello abre la posibilidad de emplear la escala visual de forma generalizada en el entorno industrial, como alternativa a las mediciones instrumentales, pues el uso de estas últimas requiere inversiones económicas que están fuera del alcance de la mayor parte de la industria.

No obstante lo analizado hasta el momento, se debe señalar que el presente estudio no propone el uso del patrón visual como único criterio para determinar la calidad de la carne. Aunque se ha documentado ampliamente que el color es un factor clave en la decisión de compra por parte del consumidor, se sabe que éste no se correlaciona con la suavidad o con la aceptación sensorial de la carne ${ }^{(4)}$. Por tanto, si se pretende evaluar la calidad de las canales, será preciso complementar la evaluación del color con la de otros atributos de calidad (ej. pH final, madurez fisiológica de los animales, marmoleo, entre otros) ${ }^{(7)}$. Adicionalmente, la escala que aquí se presenta se basa en mediciones realizadas a las $24 \mathrm{~h}$ post mortem. Por tanto, su aplicación en el contexto de la cadena de distribución podría no ser del todo consistente, pues la temperatura de almacenamiento, el tipo de empaque, la bioquímica del músculo, entre otros factores, pueden modificar el color de la carne y la estabilidad del $\operatorname{mismo}^{(11,37)}$.

Por otra parte, aunque es posible optar por el uso exclusivo de la medición instrumental para evaluar el color, se deben tomar ciertas precauciones. En primer lugar, los valores de L* típicos que se reportan para cada categoría se midieron con un espectrofotómetro cuya configuración (apertura del puerto, geometría, iluminante, etc.) puede ser diferente a la de otros equipos. Por tanto, el uso de otros instrumentos o configuraciones distintas a las aquí empleadas pueden hacer variar los resultados. 
A pesar de lo anterior, la escala desarrollada puede ser muy útil para estimar, desde los rastros, si la apariencia de la carne pudiera repercutir positiva o negativamente en la decisión de compra por parte de los clientes. Además, su empleo puede facilitar una comunicación más eficiente, por el uso de un descriptor técnico objetivo, al momento de comercializar la carne. Particularmente, la escala demostró funcionar muy bien en lo que respecta a la identificación de carne con apariencia de corte oscuro, cuya temprana detección en los rastros es de gran importancia económica.

\section{Conclusiones e implicaciones}

La escala descriptiva desarrollada en este estudio provee ilustraciones típicas de la apariencia que puede tomar la carne de bovino en México, así como de los intervalos de L* asociados con cada una de ellas. La concurrencia de criterios visuales e instrumentales en la herramienta permite su puesta en práctica de manera versátil, ya sea con paneles sensoriales, con mediciones instrumentales o mediante la combinación de ambos. La escala está concebida como herramienta para la evaluación del color en el rastro, a las $24 \mathrm{~h}$ post mortem y ha mostrado un excelente desempeño para la detección de carne con apariencia de corte oscuro en pruebas de validación realizadas en el entorno industrial. En empresas que realicen evaluación de canales, esta herramienta podría integrarse como un criterio más para definir la calidad de las mismas.

\section{Agradecimientos}

Este estudio fue realizado con recursos del fondo sectorial SAGARPA-CONACYT, proyecto 109127. Los autores agradecen al profesor Melvin C. Hunt, por su asistencia técnica para la revisión y puesta a punto de la metodología de trabajo empleada en el presente trabajo

\section{Literatura citada:}

1. Wulf D, Wise W. Measuring muscle color on beef carcass using the L*a*b* color space. J Anim Sci 1999;77(9):2418-2427.

2. Mancini RA, Hunt MC. Current research in meat color. Meat Sci 2005;71(1):100-21.

3. Leyva-García IA, Figuerosa-Saavedra F, Sánchez-López E, Pérez-Linares C, BarrerasSerrano A. Impacto económico de la presencia de carne DFD en una planta de sacrificio Tipo Inspección Federal (TIF). Arch Med Vet 2012;44(1):39-42.

4. Troy DJ, Kerry JP. Consumer perception and the role of science in the meat industry. Meat Sci 2010;86(1):214-226. 
5. Prieto N, Lopez-Campos O, Suman SP, Uttaro B, Rodas-Gonzalez A, Aalhus JL. Exploring innovative possibilities of recovering the value of dark-cutting beef in the Canadian grading system. Meat Sci 2018;137(1):77-84.

6. Aus-Meat. Handbook of Australian meat, 7th ed. Australia: South Brisbane; 2005.

7. Aalhus JL, López-Campos Ó, Prieto N, Rodas-González A, Dugan MER, Uttaro B, Juárez M. Review: Canadian beef grading - Opportunities to identify carcass and meat quality traits valued by consumers. Can J Anim Sci 2014;94(4):545-556.

8. JMGA. Japan Meat Grading Association. Beef carcass grading standard. http://wagyu.org/breed-info/meat-grading/. Accessed May 5, 2018.

9. Tatum D. Beef grading. National Cattlemen's Beef Association. https://www.beefresearch.org/CMDocs/BeefResearch/PE_Fact_Sheets/Beef_Grading. pdf. Accessed May 4, 2018.

10. Carpenter CE, Cornforth DP, Whittier D. Consumer preferences for beef color and packaging did not affect eating satisfaction. Meat Sci 2001;57(4):359-363.

11. King DA, Shackelford SD, Wheeler TL. Relative contributions of animal and muscle effects to variation in beef lean color stability. J Anim Sci 2011;89(5):1434-51.

12. King DA, Shackelford SD, Kuehn LA, Kemp CM, Rodriguez AB, Thallman RM, Wheeler TL. Contribution of genetic influences to animal-to-animal variation in myoglobin content and beef lean color stability. J Anim Sci 2010;88(3):1160-1167.

13. Chávez A, Pérez E, Rubio MS, Méndez RD, Delgado EJ, Díaz D. Chemical composition and cooking properties of beef forequarter muscles of Mexican cattle from different genotypes. Meat Sci 2012;91(2):160-164.

14. SAGARPA. ACUERDO por el que se dan a conocer las Reglas de Operación del Programa de Fomento Ganadero de la Secretaría de Agricultura, Ganadería, Desarrollo Rural, Pesca y Alimentación.

http://dof.gob.mx/nota_detalle.php?codigo=5327093\&fecha=18/12/2013. Consultado 4 May, 2018.

15. FAOSTAT. Food Balance Sheets. Food and Agricultural Organization of the United Nations, Statistics Division. http://faostat3.fao.org/download/FB/FBS/E. Accessed May 6, 2018

16. SCFI. NMX-FF- 078-SCFI-2002. Productos pecuarios - carne de bovino en canal clasificación (cancela a la NMX-FF-078-1991). http://www.economianmx.gob.mx/normas/nmx/2002/nmx-ff-078-scfi-2002.pdf. Consultado 5 May, 2018. 
17. Girolami A, Napolitano F, Faraone D, Braghieri A. Measurement of meat color using a computer vision system. Meat Sci 2013;93(1):111-118.

18. Pérez LC, Figueroa SF, Barreras SA. Relationship between management factors and the occurrence of DFD meat in cattle. J Anim Vet Adv 2006;5(7):578-581.

19. Peréz LC, Figueroa-Saavedra F, Barreras-Serrano A. Management factors associated to DFD meat in bovine on desertic climate. Arch Zoot 2008;57(220):545-547.

20. Torrescano UG, Sánchez EA, Vásquez PM, Paz PR, Pardo GD. Characterization of bovine carcasses and meat from animals fattened in Central Sonora. Rev Mex Cienc Pecu 2010;1(2):157-168.

21. Miranda-de la Lama GC, Leyva IG, Barreras-Serrano A, Perez-Linares C, SánchezLópez E, et al. Assessment of cattle welfare at a commercial slaughter plant in the northwest of Mexico. Trop Anim Health Prod 2012;44(3):497-504.

22. Zorrilla-Rios JM, Lancaster PA, Goad CL, Horn GW, Hilton GG, Galindo JG. Quality evaluation of beef carcasses produced under tropical conditions of México. J Anim Sci 2013;91(1):477-482.

23. Mendez RD, Meza CO, Berruecos JM, Garces P, Delgado EJ, Rubio MS. A survey of beef carcass quality and quantity attributes in Mexico. J Anim Sci 2009;87(11):37823790.

24. AMSA. Meat color measurement guidelines. American Meat Science Association. http://www.meatscience.org/publications-resources/printed-publications/amsa-meatcolor-measurement-guidelines. Accessed May 5, 2018.

25. Abril M, Campo MM, Onenc A, Sanudo C, Alberti P, Negueruela AI. Beef colour evolution as a function of ultimate $\mathrm{pH}$. Meat Sci 2001;58(1):69-78.

26. Costell E, Durán L. El análisis sensorial en el control de calidad de los alimentos. III. Planificación, selección de jueces y diseño estadístico. Rev Agroquím Tecnol Aliment $1981 ; 21(4): 454-470$.

27. Fernández-Vázquez R, Stinco CM, Hernanz D, Heredia FJ, Vicario IM. Colour training and colour differences thresholds in orange juice. Food Qual Prefer 2013;30(2):320-327.

28. Martínez JA, Melgosa M, Pérez MM, Hita E, Negueruela AI. Note. Visual and instrumental color evaluation in red wines. Food Sci Technol Int 2001;7(5):439-444.

29. Holman BWB, Mao Y, Coombs CEO, van de Ven RJ, Hopkins DL. Relationship between colorimetric (instrumental) evaluation and consumer-defined beef colour acceptability. Meat Sci 2016;121(1):104-106. 
30. Goñi V, Indurain G, Hernández B, Beriain MJ. Measuring muscle color in beef using an instrumental method versus visual color scales. J Muscle Foods 2008;19(2):209-221.

31. Adsitey F, Nurul H. Pale soft exudative (PSE) and dark firm dry (DFD) meats: causes and measures to reduce these incidences - a mini review. Int J Food Res 2011;18:11-20.

32. Aalhus JL, Best DR, Murray AC, Jones SDM. A comparison of the quality characteristics of pale, soft and exudative beef and pork. J Muscle Foods 1998;9:267-280.

33. Ngapo TM, Brana Varela D, Rubio Lozano MS. Mexican consumers at the point of meat purchase. Beef choice. Meat Sci 2017;134:34-43.

34. Gagaoua M, Picard B, Monteils V. Associations among animal, carcass, muscle characteristics, and fresh meat color traits in Charolais cattle. Meat Sci 2018;140:145156.

35. Holdstock J, Aalhus JL, Uttaro BA, Lopez-Campos O, Larsen IL, Bruce HL. The impact of ultimate $\mathrm{pH}$ on muscle characteristics and sensory attributes of the longissimus thoracis within the dark cutting (Canada B4) beef carcass grade. Meat Sci 2014;98(4):842-849.

36. SIAP. Resumen estatal pecuario, sector porcino. Producción, precio, valor y peso de ganado en pie 2014. http://www.siap.gob.mx/ganaderia-resumen-estatal-pecuario/. Consultado 5 May, 2018.

37. de Huidobro FR, Miguel E, Blazquez B, Onega E. A comparison between two methods (Warner-Bratzler and texture profile analysis) for testing either raw meat or cooked meat. Meat Sci 2005;69(3):527-36. 
(Información suplementaria)

Cuadro S1: Modelos de predicción de la categoría visual, ensayados en una estrategia de selección paso a paso, utilizando la categoría visual como variable dependiente y las variables instrumentales del color y sus interacciones, como variables explicativas $(n=1165)$ en el procedimiento de Modelos Lineales Generalizados de Statgraphics XV Centurion

\begin{tabular}{llll}
\hline $\begin{array}{l}\text { Variables explicativas incluidas en el } \\
\text { modelo }\end{array}$ & $\mathbf{E S}^{\mathbf{1}}$ & $\boldsymbol{P}$ & $\mathbf{R}^{\mathbf{2}}$ \\
\hline $\mathrm{L}^{*}$ & 0.40 & $<0.0001$ & 0.9145 \\
$\mathrm{a}^{*}$ & 1.22 & $<0.0001$ & 0.2134 \\
$\mathrm{~b}^{*}$ & 1.13 & $<0.0001$ & 0.3269 \\
$\mathrm{C}^{*}$ & 1.18 & $<0.0001$ & 0.2651 \\
$\mathrm{~h}^{*}$ & 1.05 & $<0.0001$ & 0.4172 \\
$\mathrm{~L}^{*}, \mathrm{a}^{*}, \mathrm{~b}^{*}, \mathrm{C}^{*}, \mathrm{~h}^{*}$ & 0.40 & $<0.0001$ & 0.9168 \\
$\mathrm{~L}^{*}, \mathrm{a}^{*}, \mathrm{~b}^{*}, \mathrm{C}^{*}$ & 0.40 & $<0.0001$ & 0.9153 \\
$\mathrm{~L}, \mathrm{a}^{*}, \mathrm{~b}^{*}\left(\mathrm{~L}^{*} \mathrm{x} \mathrm{a} \mathrm{a}^{*} \mathrm{x} \mathrm{b}\right)$ & 0.40 & $<0.0001$ & 0.9163 \\
$\mathrm{~L}^{*}, \mathrm{a}^{*},\left(\mathrm{~L}^{*} \mathrm{x} \mathrm{a}\right)$ & 0.40 & $<0.0001$ & 0.9165 \\
$\mathrm{~L}^{*}, \mathrm{~b}^{*},\left(\mathrm{~L}^{*} \mathrm{x} \mathrm{b}\right)$ & 0.40 & $<0.0001$ & 0.9159 \\
$\mathrm{~L}^{*}, \mathrm{C}^{*},\left(\mathrm{~L}^{*} \mathrm{x} \mathrm{C} *\right)$ & 0.40 & $<0.0001$ & 0.9160 \\
$\mathrm{~L}^{*}, \mathrm{~h}^{*},\left(\mathrm{~L}^{*} \mathrm{x} \mathrm{h}\right)$ & 0.40 & $<0.0001$ & 0.9156 \\
\hline
\end{tabular}

${ }^{1}$ Error estándar del estimador.

Cuadro S2: Valor F y nivel de significación estadística $(P)$ del ANOVA realizado con los datos de calificación del color emitidos por cada juez entrenado $(n=36)$ en muestras de carne con apariencia rojo pálida, normal, $\mathrm{CO}$ moderada y $\mathrm{CO}$ extrema

\begin{tabular}{lll}
\hline Juez & FANOVA & $\boldsymbol{P}$ \\
\hline 1 & 193.3 & $<0.0001$ \\
2 & 139.1 & $<0.0001$ \\
3 & 138.3 & $<0.0001$ \\
4 & 116.5 & $<0.0001$ \\
5 & 103.9 & $<0.0001$ \\
6 & 91.9 & $<0.0001$ \\
\hline
\end{tabular}


Cuadro S3: ANOVA realizado con los datos de calificación del color emitidos por el panel sensorial entrenado en muestras de carne con apariencia rojo pálida, normal, CO moderada y CO extrema

\begin{tabular}{llllll}
\hline Fuente & $\begin{array}{l}\text { Suma de } \\
\text { cuadrados }\end{array}$ & GL $^{\mathbf{1}}$ & Cuadrado medio & Razón-F & Valor-P \\
\hline Entre grupos & 141.022 & 3 & 47.0074 & 639.52 & 0.0000 \\
Intra grupos & 7.64444 & 104 & 0.0735043 & \\
Total (Corr.) & 148.667 & 107 & & \\
\hline \multicolumn{5}{c}{${ }^{1}$ Grados de libertad. }
\end{tabular}

Cuadro S4: ANOVA multifactorial ${ }^{1}$ para la calificación del color

\begin{tabular}{lll}
\hline Efecto & Razón-F & Valor-P \\
\hline Juez & 0.95 & 0.4491 \\
Muestra & 7.04 & 0.0002 \\
Sesión & 0.89 & 0.4919 \\
Juez x Muestra & 0.86 & 0.6126 \\
Juez x Sesión & 0.68 & 0.8701 \\
Muestra x Sesión & 1.03 & 0.4254 \\
Juez x Muestra x Sesión & 0.92 & 0.9860 \\
\hline
\end{tabular}

